\title{
Survival Analysis of Upper Arm Replantation After Avulsion Injuries
}

\author{
Xiaolong Du, MD'; Yafei Liu, MD'; Xuehai Ou, MD'; Yongming Xiong, MD, PhD²; Fei Cong, MD'; \\ Yang Liu, MD'; Tao Song, MD* \\ 1Department of Orthopaedics, Xi'an Honghui Hospital, Xi'an Jiaotong University, Xi'an, Shaanxi, China \\ 2Institute of Endemic Diseases, Department of Medicine, Xi'an Jiaotong University, Xi'an, Shaanxi, China
}

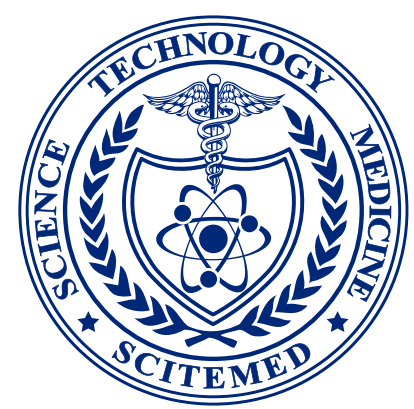

\begin{abstract}
Background: Avulsion injuries and replantation of the upper arm are particularly challenging in the field of traumatic microsurgery. At present, the functional recovery of the avulsion injuries upper arm after the replantation is generally not ideal enough, and there is no guideline for the surgeries. The aim of this study was to analyze the causes of failure of the upper arm replantation for avulsion injuries, summarize the upper arm replantation's indications, and improve the replantation methods.

Methods: From January 2014 to January 2016, our department had treated 15 cases of trauma caused upper arm avulsion amputation injuries. All the injured arms had been replanted. We conducted rapid debridement, simple and effective external fixation, or internal fixation, and used microsurgical methods to repair blood vessels and nerves. After the surgery, the patients were prescribed medicines such as anti-inflammatory, anti-spasm, prophylactic antibiotics, anticoagulation, rehydration, and analgesic treatment. We also monitored patients' blood supply situation closely.

Results: The 15 cases of the upper arm replantation for avulsion injuries resulted in a 100\% postoperative patency rate, $73.3 \%$ skin necrosis rate, $60 \%$ muscle necrosis rate, and $20 \%$ amputation rate. Of all the cases, three cases were required to remove the replant limb due to unstable vital signs, acute renal failure, and major artery bleeding during the debridement. The average of the follow-up was two years. After one year of the operation, the replanted upper arm's function was assessed according to the Function score of upper limb replantation of Hand Surgery Society of Chinese Medical Association. The average score was 25.2 points, which was classified as poor.

Conclusion: The upper arm avulsion injuries after trauma, which should have been treated with microsurgery, remains a challenging problem. There has been a lack of formed upper extremity replantation guide, and the patients are often hemodynamically unstable with multiple traumas and combined injuries, resulting in a high risk of failure. The long ischemia time often attributes to surgical failures and amputation. Skilled microsurgical techniques may improve the success rate of replantation.
\end{abstract}

\section{INTRODUCTION}

Replantation of the avulsed upper arm is particularly challenging in the field of traumatic microsurgery. It is usually a high-level injury, severe soft tissue inju$r y$, and often combined with severe hemorrhagic shock and other complicated associate injuries which make the replantation a great risk. At present, the functional recovery of the avulsion injuries over upper arm after the replantation is generally not satisfied enough, and there is no guideline for the surgeries. There is still much controversy in this field [1,2].

On the other hand, the upper limb prostheses are not only expensive but also difficult to meet the needs of patients' functional requirements. The upper limb prostheses are less useful than the lower extremity prostheses and the importance of replantation is still irreplaceable [3]. How to further clarify the indications for surgery, improve surgical techniques, and promote full recovery of patients with upper extremity function are still important issues in the field of limb replantation [4]. From January 2014 to January 2016, 15 cases of upper extremity avulsion injuries were treated in our department. Among them, three patients were amputated after the replantation surgery. The indications, surgical techniques, and rehabilitation methods of the replantation of the upper arm were discussed in this article.

\section{METHODS}

\section{General Information}

There were 15 cases in this study, eleven males and four females. They were between 19-57 years old, with an average age of 35.8 years. Causes of injury include eight cases of machine injury, four cases of car accident, two cases of belt pulley injury, and one case of mixer injury. Injury sites include six cases of elbow, six cases of middle arm, and three cases of proximal arm. The ischemic time was 4-12 hours, an average of 6.26 hours. All patients suffered vascular and nerve defects. The defect length was 4-7 cm. Ten cases were performed shortening replantation and five cases were performed vascular graft replantation. Patients summary is shown in Table 1.

\section{Surgical Methods}

All the patients were operated under general anesthesia. Surgeons were divided into two teams and performed the following procedures concurrently. One team focused on the debridement, dissection, and marked the main vessels and nerves of the distal limb; and another team performed the same procedure on the proximal limb. The method of the surgery either shorten the limb for replant or transplant the vein graft to bridge the defect was decided according to the vessel defects, muscle, skin, and other soft tissue defects. If the fracture is comminuted and the skin, muscle defect is more than $5 \mathrm{~cm}$, shorten limb replantation is selected. On the contrary, free blood vessel transplantation was chosen to replant the limbs. The average length of shortening of upper arm is $5 \mathrm{~cm}$. For shortening replanted group, distal and proximal humeral shortening osteotomy were performed with the use of wire saw, then, a plate or external fixator was utilized to fix the broken ends. Under the microscope, the brachial artery and accompanying veins, cephalic vein, basilic vein were anastomosed, radial nerve, ulnar nerve, median nerve, and musculocutaneous nerve were copated, muscles were sutured to cover the vascular and nerves, placed drainage tube, and the skin closed. For non-shortening replanted group, the vein grafts and nerve graft required to bridge the defect. First, a plate or external fixator was used to quickly fix the broken ends, harvested the great saphenous vein from the contralateral lower limb, then bridged the brachial artery and anastomosed the major nerves, harvested the 


\begin{tabular}{|c|c|c|c|c|c|c|c|c|}
\hline No. & Gender & Age & Level of injuries & Ischemia time & Total operating time & Complications & $\begin{array}{l}\text { No. of subsequent surgeries } \\
\text { after the replantation }\end{array}$ & Score of the limb* \\
\hline 1 & Female & 48 & Proximal arm & 4 hours & 6 hours & Skin necrosis & 1 & 35 \\
\hline 2 & Male & 22 & Shoulder, elbow & 7 hours & 14 hours & Skin necrosis, amputation & 1 & 0 \\
\hline 3 & Male & 40 & Elbow & 12 hours & 6 hours & Skin necrosis, amputation & 2 & 0 \\
\hline 4 & Male & 44 & Middle arm & 4 hours & 4 hours & No & 0 & 59 \\
\hline 5 & Female & 39 & Middle arm & 6 hours & 6.5 hours & Skin necrosis, muscle necrosis & 2 & 31 \\
\hline 6 & Male & 57 & Elbow & 5 hours & 5 hours & Skin necrosis, muscle necrosis & 1 & 25 \\
\hline 7 & Male & 33 & Proximal arm & 4 hours & 4 hours & No & 0 & 38 \\
\hline 8 & Male & 19 & Middle arm & 6 hours & 6 hours & Skin necrosis, muscle necrosis & 4 & 24 \\
\hline 9 & Female & 43 & Elbow & 6 hours & 8 hours & Skin necrosis, muscle necrosis & 5 & 22 \\
\hline 10 & Male & 31 & Elbow & 4 hours & 5 hours & Skin necrosis, muscle necrosis & 1 & 31 \\
\hline 11 & Male & 35 & Middle arm & 5 hours & 4 hours & Muscle necrosis & 2 & 30 \\
\hline 12 & Male & 45 & Proximal arm & 8 hours & 7 hours & Skin necrosis, muscle necrosis & 4 & 20 \\
\hline 13 & Female & 28 & Elbow & 7 hours & 6 hours & Skin necrosis, muscle necrosis & 4 & 18 \\
\hline 14 & Male & 30 & Middle arm & 6 hours & 4 hours & Muscle necrosis & 3 & 45 \\
\hline 15 & Male & 23 & Middle arm & 9 hours & 6 hours & Skin necrosis, amputation & 2 & 0 \\
\hline
\end{tabular}

"The score of the limbs are according to the Function score of upper limb replantation of Hand Surgery Society of Chinese Medical Association.

sural nerve from the contralateral lower limb, then bridged the major nerves. And anastomosed the cephalic vein and basilic vein, sutured muscle to cover the vessels and nerves, placed drainage tube, and sutured the skin. Skin necrosis and muscle necrosis were found to be the most common complications after the initial operation. Debridement and skin grafting were usually performed, some cases had vascular embolism, we performed the vessel transplantation for the patient.

\section{Postoperative Management and Rehabilitation}

After the replantation surgery, all patients were prescribed medicines including anti-inflammatory, anti-coagulant, anti-vasospasm, analgesic, vasodilator, transfusion, and rehydration therapy; electrocardiography, blood oxygen saturation monitoring and blood pressure monitoring, review of the blood test, coagulation series, liver and kidney function tests, electrolytes and myocardial enzymes monitoring were also performed. The patients were completely bed ridden for seven days with limb warmth, no smoking, no mood swings, and changes in peripheral blood flow and skin temperature. After four weeks, the patients were required to move their fingers passively. After eight weeks, passive wrist and hand functional exercises, active shoulder, and elbow functional exercises were practiced. Different surgeries such as functional muscle, nerve, or tendons transfers were performed according to each patient's condition during the functional recovery period. Three cases underwent nerve transfers and four cases underwent free tendons or functional muscle transfers. The four patients with elbow flexion dysfunction underwent functioning free gracilis muscle transplantation to reconstruct elbow joint function.

\section{RESULTS}

After the replantation surgery, doppler was used to explore the distal vascular patency and all the radial arteries of the patients could be detected. Vascular patency rate was $100 \%$. Eleven patients had skin necrosis after the surgery; the incidence of skin necrosis was $73.3 \%$. Nine patients had various muscle necroses; the incidence of muscle necrosis was $60 \%$. A total of three patients were amputated; the amputation rate of patients was $20 \%$. Elbow amputation is the osteotomy of the distal humerus to avoid the expand of the distal limb. One patient had avulsion of the upper arm and multiple ribs fractures. First day after the replantation surgery, the patient had elevated serum potassium, elevated myocardial enzymes, thrombocytopenia, coagulation disorders, and respiratory failure. An emergency amputation was performed; however, it did not save the patient's life. One patient with acute renal failure was also amputated. One case was 20 days of discharge who had muscle necrosis; when debridement was done, the blood vessels were ruptured, and then he was amputated. Thirteen wound secretions were cultured after the operation and the infection rate was $86.7 \%$. The patients were followed up for one to three years, with an average of two years. Upper limb function at one year after the replantation surgery was an average score of 25.2 points, which was considered poor function according to the Chinese Medical Association assessment criteria for upper limb amputation replantation (Table 2).

\section{Case Report}

A 48 years old female was presented with a right upper arm avulsion injury caused by strangulation of the machine. The patient underwent shortening replanted surgery, and the limb was survived. The skin defect over axillary area was closed by the skin grafting, the outcome was satisfactory (Figure 1).

\section{DISCUSSION}

\section{Upper Arm Avulsion and Replantation Indications}

Avulsion injuries and replantation of the upper arm are particularly challenging in the field of traumatic microsurgery. Because avulsion injuries are usually high-level, severe soft tissue injuries, and often with severe hemorrhagic shock and other complex injuries. The replantation of upper arm possesses a great risk, and its indications in our institute include age below 60 years, no heart disease, hypertension, diabetes, or other underlying diseases, warm ischemia time is less than six hours, and without chest, abdomen, or head complex injury. The mangled extremity severity score (MESS) is one of the few scoring systems to predict limb salvage outcomes of upper extremity injures. However, MESS has been tested and found not very useful in several studies. The prevailing opinion is that in the upper extremity injury, every attempt should be made to salvage the limb. Another important indication is the will of the patient and his family. The contraindications of upper arm replantation in 
Table 2. Upper Limb Replantation Function Score of Hand Surgery Society of Chinese Medical Association

\begin{tabular}{|c|c|}
\hline Range of motion (30) & Score \\
\hline \multicolumn{2}{|l|}{ Abduction of shoulder joint } \\
\hline $90^{\circ} \sim 60^{\circ}$ & 6 \\
\hline $59^{\circ} \sim 45^{\circ}$ & 5 \\
\hline $44^{\circ} \sim 30^{\circ}$ & $3 \sim 4$ \\
\hline$<30^{\circ}$ & $0 \sim 2$ \\
\hline \multicolumn{2}{|l|}{ Elbow (flexion and extension) } \\
\hline $120^{\circ} \sim 90^{\circ}$ & $7 \sim 8$ \\
\hline $89^{\circ} \sim 60^{\circ}$ & $5 \sim 6$ \\
\hline $59^{\circ} \sim 30^{\circ}$ & $3 \sim 4$ \\
\hline$<30^{\circ}$ & $0 \sim 2$ \\
\hline \multicolumn{2}{|l|}{ Wrist (flexion and extension) } \\
\hline $60^{\circ} \sim 90^{\circ}$ & $3.1 \sim 4$ \\
\hline $45^{\circ} \sim 59^{\circ}$ & $2 \sim 3$ \\
\hline $30^{\circ} \sim 44^{\circ}$ & $1.5 \sim 2$ \\
\hline$<30^{\circ}$ & $0 \sim 1.5$ \\
\hline \multicolumn{2}{|c|}{ Metacarpophalangeal joint (flexion and extension) } \\
\hline $90^{\circ} \sim 70^{\circ}$ & $4.1 \sim 5$ \\
\hline $69^{\circ} \sim 50^{\circ}$ & $3.1 \sim 4$ \\
\hline $49^{\circ} \sim 30^{\circ}$ & $2.1 \sim 3$ \\
\hline$<30^{\circ}$ & $0 \sim 1.4$ \\
\hline \multicolumn{2}{|c|}{ Proximal interphalangeal joint (flexion and extension) } \\
\hline $100^{\circ} \sim 80^{\circ}$ & 3.1 4 \\
\hline $79^{\circ} \sim 60^{\circ}$ & $2.1 \sim 3$ \\
\hline $59^{\circ} \sim 30^{\circ}$ & $1.5 \sim 2$ \\
\hline$<30^{\circ}$ & $0 \sim 1.4$ \\
\hline \multicolumn{2}{|c|}{ Distal interphalangeal joint (flexion and extension) } \\
\hline $45^{\circ} \sim 30^{\circ}$ & $2.1 \sim 3$ \\
\hline $29^{\circ} \sim 20^{\circ}$ & $1.1 \sim 2$ \\
\hline $19^{\circ} \sim 15^{\circ}$ & 1 \\
\hline$<15^{\circ}$ & 0 \\
\hline \multicolumn{2}{|l|}{ Muscle strength (20) } \\
\hline$>\mathrm{M} 4$ & $17 \sim 20$ \\
\hline M4 & $13 \sim 16$ \\
\hline M3 & $8 \sim 12$ \\
\hline$<\mathrm{M} 2$ & $0 \sim 7$ \\
\hline \multicolumn{2}{|l|}{ Sensation (20) } \\
\hline S4 & $16 \sim 20$ \\
\hline S3+ & 12 15 \\
\hline S3 & $8 \sim 11$ \\
\hline$<\mathrm{S} 2$ & $0 \sim 7$ \\
\hline \multicolumn{2}{|l|}{ Appearance (10) } \\
\hline Normal or slightly atrophic & $8.1 \sim 10$ \\
\hline Slightly atrophic & $6.1 \sim 8$ \\
\hline Moderate atrophy & $3 \sim 6$ \\
\hline Apparent atrophy & $0 \sim 2$ \\
\hline \multicolumn{2}{|l|}{ Residual symptoms (10) } \\
\hline No numbness, pain or other discomfort & 10 \\
\hline Mild numbness, pain or other discomfort & 7 \\
\hline Numbness, pain or other discomfort & 3 \\
\hline Pain, allergies or becoming a burden & 0 \\
\hline \multicolumn{2}{|l|}{ Work situation (10) } \\
\hline Returned to the former job & 10 \\
\hline Engage in light work & 7 \\
\hline Able to meet the needs of daily life & 3 \\
\hline No practical function & 0 \\
\hline \multicolumn{2}{|l|}{ Evaluation method } \\
\hline Excellent (100 80) & Good (79 60) \\
\hline Negative (59 40) & Terrible (<39) \\
\hline
\end{tabular}
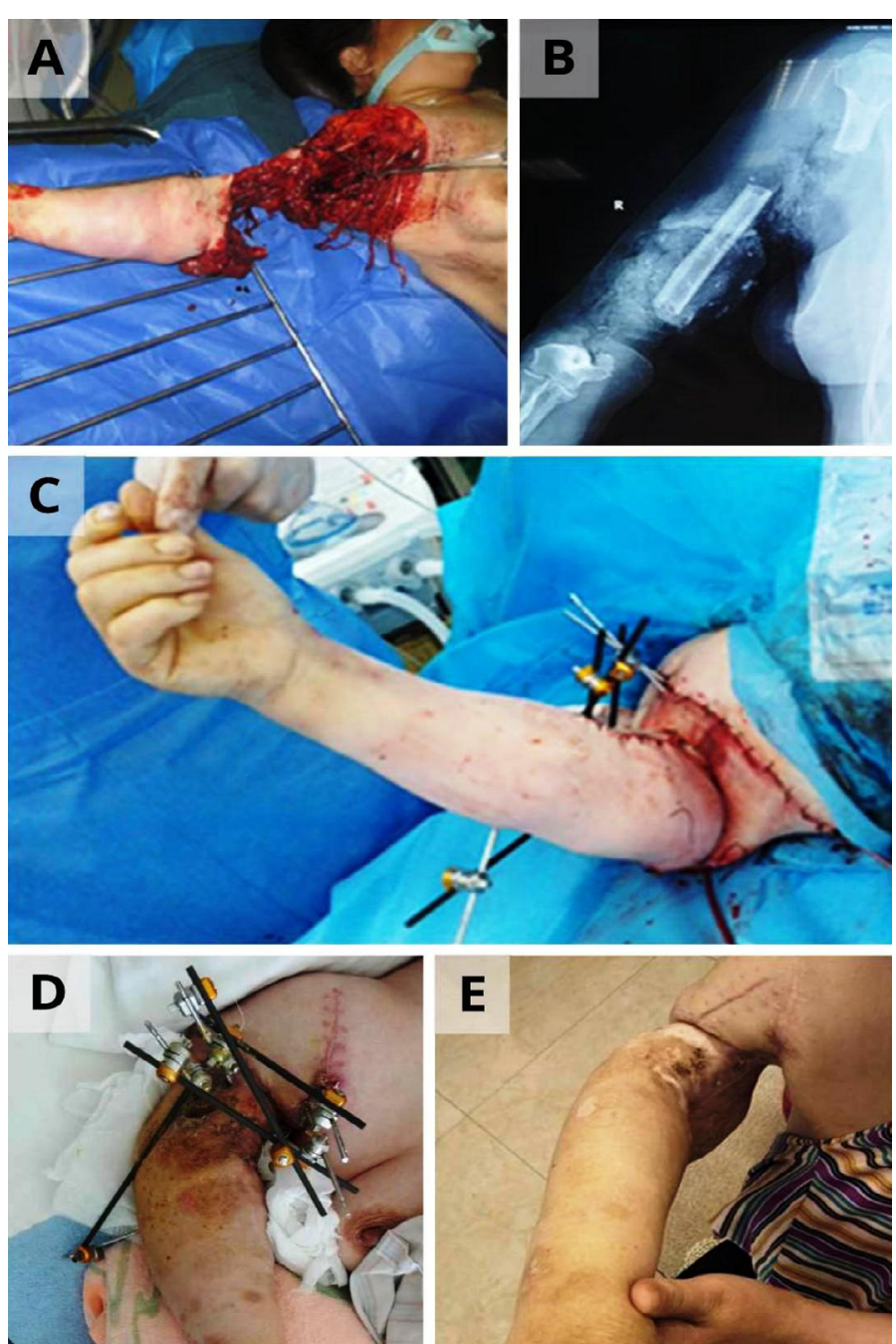

Figure 1. (A) Preoperative appearance. (B) Preoperative X-ray. (C) Postoperative appearance. (D) Four weeks after the replantation. (E) One year after the replantation.

our institute include severe proximal arm avulsions or shoulder disarticulation with proximal nerve avulsion from brachial plexus nerves or roots [1].

\section{Survival Analysis}

After the replantation surgery of severed upper arm avulsion injury, the affected limb had a high rate of vascular patency; because of the thick upper arm vasculature, the collateral circulation was more abundant. However, $86.7 \%$ of the cases required secondary surgeries; the patients with muscle necrosis and infection underwent multiple debridement, the patients with skin necrosis received skin grafting, the patients with vascular embolism was performed vascular exploration, and the patients with limb necrosis and unstable vital signs required an emergency amputation. Due to severe skin and soft tissue contusion, the incidence of postoperative skin necrosis was $73.3 \%$. The incidence of muscle necrosis was also high, $60 \%$. Muscle ischemia-resistant time was 6 hours, for the patient injured to the recanalization of the blood vessels usually needed more than 6 hours. After recanalization, although the limbs could be saved, the muscle necrosis was inevitable. The consequent wound infection was also inevitable. Among the group of 15 cases of replantation patients, three patients were performed the amputation surgery, the amputation rate was $20 \%$. Among the three patients who received amputations. One patient died of upper limb avulsion after the replantation surgery. The patient was male, 25 years old, who was admitted to the hospital with bilateral upper extremities avulsion injuries, bilateral clavicle fracture, bilateral scapular fracture, and bilateral multiple rib fractures. The patient had elevated serum 
potassium, elevated myocardial enzymes, thrombocytopenia, coagulation disorders, and respiratory failure on the first day after the replantation surgery. We did the emergency amputation, but the patient was still dead. One patient had acute renal failure, creatine kinase was $17000 \mathrm{u} / \mathrm{L}$, persistent fever, confusion, and we did the emergency amputation surgery for him. One patient was discharged 10 days after the operation and transferred to a lower hospital for treatment. During the procedure of dressing change, the brachial artery was injured. On the 20th day after the operation, the limb of the patient with devascularization was replanted and returned to our hospital again. After exploration, muscle necrosis and peripheral devascularization were found in the affected limb, and finally, amputation was performed. With the development of microsurgery, the success rate of vascular anastomosis had been very high in traumatic micro-orthopedics in the case of the vascular graft after severed wounds in the large limbs. However, patients often suffered more injuries. To increase the success of the replantation, at the beginning of the surgery, we used a tube to connect the main artery for shunting to reduce the ischemic time of the severed limb.

\section{Factors Influencing the Survival Rate}

For patients with stable vital signs, no fatal composite injury was the primary condition for limb survival. On the other hand, one patient in this study had severe upper arm avulsion, and other injuries include bilateral scapula, clavicle fractures, and multiple fractures of the ribs. The patient died from severe complications after the replantation surgery. Preoperative detailed physical examination was necessary to avoid unfavorable postoperative outcomes, and the patients needed relevant departments' consultation; and lifesaving should be considered priority before limb salvage. The quality of vascular anastomosis was also an important factor for the survival of replantation limbs. Because of the long segment of blood vessel contusion and avulsion, the plane of vascular injury was difficult to judge. Surgeons must judge the length of the vascular defect accurately. Before the shortening replantation or vascular graft replantation, we used catheter shunting surgery to provide the blood supply of the distal limb, which could greatly shorten the time of muscle ischemia and prevent future muscle tissue necrosis, leading to a better postoperative function recovery and significantly improving the survival rate of replantation and muscle necrosis.

\section{The Replantation Limb Function Recovery}

Patients with upper limb avulsion and replantation generally had poor functional recovery after transplantation. Although most of the patients had their nerves repaired during operation, the postoperative functional recovery was extremely limited due to the nerve avulsion. Because of the longtime of muscle ischemia after replantation of avulsion injury of the upper arm, which will lead to muscle fibrosis and even necrosis, we suggest that the catheter should be used to open the blood of the distal limb for the first time, so as to minimize the ischemic time of the distal limb. In addition, the avulsed nerve should be repaired as early as possible. If the nerve was damaged, nerve transplantation can be carried out early, so that the function of the severed limb can be restored to the greatest extent. After replantation, the early passive activities also play an important role in the recovery of limb function. The time of 4-8 weeks after the surgery is a critical period of rehabilitation. In this period, the patients may have consequences such as the upper extremity external fixation not maintaining a safe position, many scar formations, or joint stiffness which make the patients lost conditions of rehabilitation and reconstruction. During this period patients should pay attention to continue to maintain the function of the upper limb and hand. The hand and the wrist should do pas- sive activities, the shoulder and the elbow should begin to do an initiative activity with protection. Eight weeks after surgery, most of the wounds have been healed. After two years, with effective rehabilitation training, the function of the upper arm will have reconstruction. This period the patients should be unswervingly encouraged to build confidence in life and with functional reconstruction [6]. Although successful replantation is a great comfort for the patient's psychology, the loss of long-term function makes the patient's psychological expectation declining gradually. Difficult to return to the original job again, the fight against the patient's family is still huge.

\section{CONCLUSION}

Upper arm avulsion injuries after trauma remains a challenging problem, and it requires a treatment with microsurgery. There has been a lack of formed upper extremity replantation guide, and the patients are often hemodynamically unstable with multiple traumas and combined injuries, resulting in a high risk of replantation. The long waiting time from injury to inadequate blood flow often attributes to surgical failures and amputation. Skilled microsurgical techniques may improve the success rate of replantation.

\section{ARTICLE INFORMATION}

*Correspondence: Tao Song, MD. Department of Orthopaedics, Xi'an Honghui Hospital, Xi'an Jiaotong University, Xi'an, Shaanxi, China. E-mail: st0325@126.com

Received: Nov. 23, 2019; Accepted: Aug. 02, 2020; Published: Sep. 08, 2020

DOI: 10.24983 / scitemed.imj.2020.00135

Ethics Approval and Consent to Participate: The study is in accordance with the ethical standards of the 1964 Helsinki declaration and its later amendments or comparable ethical standards.

Funding: The study did not receive any specific grant from funding agencies in the public, commercial, or not-for-profit sectors.

Disclosure: This article was presented at the 10th Congress of World Society for Reconstructive Microsurgery, in Bologna, Italy, in 2019.

Conflict of Interest: The authors report no financial or other conflict of interest relevant to this article, which is the intellectual property of the authors.

Copyright @ 2020 The Authors. This is an open-access article distributed under the terms of the Creative Commons Attribution 4.0 International License (CC-BY).

\section{REFERENCES}

1. Ng WK, Kaur MN, Thoma A. Long-term outcomes of major upper extremity replantations. Plast Surg 2014;22(1):9-13.

2. Mi JY, Rui YJ, Zhao G, et al. A retrospective study of upper arm replantation. Chin J Bone and Joint 2015;12(4):946-949.

3. Lu TR. Functional evaluation of a patient 39 years after forearm replantation. Chin J Phys Med Rehabil 2006;28(12):822-824.

4. Yang RG, Hou SX, Zhang WJ, et al. Restorating function of severely impaired upper extremity using the side chest and back tissue. Chin J Orthop 2006;26(2):238-242.

5. Zeng WC, Guo HM, Wang HB, et al. Clinical experiences in severed limb replantation. Journal of Practical Orthopaedics 2015;2:184-186.

6. Yu Jun, Wang Jun, Zhu Yi. Long-term outcome analysis of the patients with complete forearm reconstruction after replantation. Chin J of Hand Surg 2015;31(1):38-40. 